\title{
Bifurcations of periodic orbits and potential stability regions in Kuiper belt dynamics
}

\author{
Thomas A. Kotoulas and George Voyatzis \\ Section of Astrophysics,Astronomy and Mechanics, Department of Physics, University of \\ Thessaloniki, GR-541 24 Thessaloniki, Greece \\ email: tkoto@skiathos.physics.auth.gr, voyatzis@auth.gr
}

\begin{abstract}
In the framework of the restricted three body problem, the resonant periodic orbits associated with the Kuiper belt dynamics are studied. Particularly, all the first, second and third order exterior mean motion resonances with Neptune located up to 50A.U. and the asymmetric resonances (beyond the 48 A.U.) are considered. We present the bifurcation points of families of periodic orbits of the planar circular problem from which families of periodic orbits are generated in the planar elliptic and in the 3D circular problem. Similarities and differences between the various resonant cases are noticed. The relation between the distribution of the bifurcation points and the population of small bodies at the particular resonances is discussed.
\end{abstract}

Keywords. Kuiper Belt, minor planets

\section{Introduction}

An interesting problem in Solar System science is the study of the dynamical evolution of small bodies beyond the orbit of Neptune, in the so called Edgeworth-Kuiper $(E-K)$ belt. More than 700 trans-Neptunian objects (TNOs) have been observed up to now and are included in the associated list of the Minor Planet Center (http://cfawww.harvard.edu/iau/ mpc.html). Most of the TNOs move in low eccentric orbits $(e \approx$ 0.1 ) but a significant portion of them shows relatively high eccentric motion. The existence of high inclined orbits is also evident. A recent review about the structure of the Edgeworth-Kuiper belt was given by Morbidelli et al. 2003.

Many numerical and theoretical works were done to investigate the dynamics of TNOs in the framework of the restricted three body problem (RTBP) (e.g. Knežević et al. (1991), Levison and Duncan (1993), Holman and Wisdom (1993), see also references in Morbidelli (1999)). Even the simplest model, namely the planar circular restricted threebody problem, reveals interesting dynamical features (Malhotra (1996)). For models of few degrees of freedom, the existence and stability of periodic orbits determine critically the phase space structure. Therefore the regions round stable periodic orbits are possible places to host TNOs revolving in regular orbits. We studied the families of periodic orbits in many resonances in E-K belt and in the present work we discuss about their properties with respect to the bifurcation points and stability of the generating periodic orbits. In Section 2 we present the main features of the families of symmetric periodic orbits in the planar circular RTBP, while in Section 3 we present the bifurcation points of each family from the planar circular to the planar elliptic and to the $3 \mathrm{D}$ circular RTBP. The resonances of the type $n / n^{\prime}=1 / q, q=2,3, \ldots$, the so-called asymmetric resonances, are discussed in Section 4. 


\section{Families of symmetric periodic orbits in the planar circular RTBP}

We consider the rotating orthogonal frame of reference $x O y$ where the Sun and the secondary body, particularly the Neptune, define the $O x$-axis. For the mass of Neptune we have chosen the value $\mu=5.178 \times 10^{-5}$ and the orbital period of Neptune around the Sun is equal to $2 \pi$. In the above rotating frame, the system has families of symmetric periodic orbits $(y(0)=0, \dot{x}(0)=0)$ classified in two different kinds:

- Families of circular orbits (first kind) : The periodic orbits correspond to nearly circular orbits for the small body. The ratio $n / n^{\prime}$ varies along each family.

- Resonant Families (second kind) : The periodic orbits correspond to elliptic orbits for the small body. The eccentricity increases along the family but the ratio $n / n^{\prime}$ is almost constant and rational $n / n^{\prime} \approx p / q, p, q \in Z$.

For the exterior resonances, studied in this paper, it is $q>p$ and the difference $q-p$ defines the order of the resonance. The multiplicity of a periodic orbit is defined as the number of crosses of the orbit with the axis $y=0$ in the same direction $(\dot{y}>0$ or $\dot{y}<0)$ in a period. For each resonance there exist two different families (I and II) differing in phase. On family I the small body is initially at perihelion and on family II it is at aphelion. Both of them originate from the circular family. For the high order resonances (e.g. 1/3, $3 / 5,1 / 4,4 / 7)$ the continuation of the family of the circular orbits is possible for $\mu>0$ and the resonant families bifurcate from the circular family where $n / n^{\prime}=p / q$. For the first order resonances (e.g. $1 / 2,2 / 3,3 / 4,4 / 5)$ the above mentioned continuation is not possible (Hadjidemetriou and Ichtiaroglou (1984)). The resonant families join smoothly the circular family, which shows a gap at the position of the first order resonance for $\mu \neq 0$. A detailed study on the $1 / 2,2 / 3$ and $3 / 4$ resonant families is given in Kotoulas and Hadjidemetriou (2002).

In all resonances, family I starts as unstable; a collision orbit with Neptune takes place at a specific value of eccentricity where the relation $a(1-e) \approx 1$ holds. After the collision orbit the family I becomes stable and the multiplicity increases by one. In all resonances except for the asymmetric ones, family II is stable except for a small area in which a collision between the small body and Neptune occurs. Along both families the eccentricity increases starting from zero and we stopped following the families because of numerical difficulties at very high values where collision with Sun takes place.

\section{Bifurcation points}

\subsection{Bifurcations from planar circular RTBP to planar elliptic RTBP}

In the elliptic problem, where the eccentricity of secondary body is $e^{\prime} \neq 0$, the families of periodic orbits bifurcate from the resonant families of periodic orbits of the circular problem $\left(e^{\prime}=0\right)$ at those points where the period is a multiple of $2 \pi$. The eccentricity values that correspond to the bifurcation points from the planar circular to planar elliptic problem are shown in Table 1 and presented in the $a-e$ plane in Fig. 1a. Two pairs of resonant families bifurcate from each point and are continued for $e^{\prime}>0$. We call them as $E_{p}$ and $E_{a}$. In family $E_{p}$ Neptune is initially at perihelion and in family $E_{a}$ it is initially at aphelion. In most of the resonant cases the family $E_{p}$ is stable while the family $E_{a}$ is unstable. In Table 1 the bifurcation points are presented and the symbols $S$ and $U$ denote stability and instability respectively of the families bifurcating from these points (the first symbol refers to family $E_{p}$ and the second one to $E_{a}$ ).

For the particular value $e^{\prime}=0.00912$ (eccentricity of Neptune's orbit) the isolated periodic orbits correspond to eccentricity values which are slightly different of that obtained for $e^{\prime}=0$. The position of such periodic orbits in the $a-e$ plane are shown in Fig. 2 
Table 1. Eccentricity values of bifurcation points (Bfn) from the planar circular to the planar elliptic RTBP. The symbols $\mathrm{S}$ and $\mathrm{U}$ denote the stability type. The first symbol refers to the generated family $E_{p}$ and the second one to family $E_{a}$.

\begin{tabular}{ccccccc}
\hline Resonance & $a_{\text {res }}$ (A.U.) & Period & Bf1 & Bf2 & Bf3 & Bf4 \\
\hline $2 / 3$ & 39.40 & $6 \pi$ & $0.469 \mathrm{SU}$ & & & \\
$3 / 4$ & 36.41 & $8 \pi$ & $0.329 \mathrm{SU}$ & & & \\
$4 / 5$ & 34.88 & $10 \pi$ & $0.253 \mathrm{SU}$ & $0.871 \mathrm{SU}$ & & \\
$5 / 6$ & 33.95 & $12 \pi$ & $0.205 \mathrm{SU}$ & $0.749 \mathrm{SU}$ & & \\
$6 / 7$ & 33.31 & $14 \pi$ & $0.172 \mathrm{UU}$ & $0.649 \mathrm{SU}$ & $0.960 \mathrm{SU}$ & \\
$3 / 5$ & 42.26 & $10 \pi$ & $0.427 \mathrm{US}$ & $0.800 \mathrm{SU}$ & & \\
$5 / 7$ & 37.62 & $14 \pi$ & $0.278 \mathrm{SU}$ & $0.562 \mathrm{SU}$ & $0.778 \mathrm{US}$ & $0.936 \mathrm{SU}$ \\
$7 / 9$ & 35.54 & $18 \pi$ & $0.203 \mathrm{SU}$ & $0.427 \mathrm{SU}$ & $0.606 \mathrm{US}$ & $0.766 \mathrm{SU}$ \\
$4 / 7$ & 43.65 & $14 \pi$ & $0.027 \mathrm{UU}$ & $0.400 \mathrm{UU}$ & $0.900 \mathrm{SU}$ & \\
$5 / 8$ & 41.12 & $16 \pi$ & $0.029 \mathrm{SU}$ & $0.335 \mathrm{US}$ & $0.800 \mathrm{SU}$ & \\
$7 / 10$ & 38.13 & $20 \pi$ & $0.025 \mathrm{SU}$ & $0.249 \mathrm{US}$ & $0.905 \mathrm{SU}$ & \\
\hline
\end{tabular}

Table 2. Eccentricity values of bifurcation points ( $\mathrm{Bf} n$ ) from planar to $3 \mathrm{D}$ circular RTBP. The character A or B denotes the symmetry type (see the text) of the orbits of the bifurcating family and the character $\mathrm{S}$ or $\mathrm{U}$ denotes the corresponding stability. The $3 / 5$ resonant bifurcations indicated by an asterisk show exceptional characteristics (see the text).

\begin{tabular}{ccccccccc}
\hline Res. & Bf1 & Bf2 & Bf3 & Bf4 & Bf5 & Bf6 & Bf7 & Bf8 \\
\hline $2 / 3$ & $0.421 \mathrm{AS}$ & $0.450 \mathrm{BU}$ & $0.968 \mathrm{BU}$ & & & & & \\
$3 / 4$ & $0.291 \mathrm{AS}$ & $0.307 \mathrm{BU}$ & $0.663 \mathrm{AU}$ & $0.753 \mathrm{AU}$ & $0.767 \mathrm{BS}$ & & & \\
$4 / 5$ & $0.222 \mathrm{AS}$ & $0.233 \mathrm{BU}$ & $0.624 \mathrm{BU}$ & $0.729 \mathrm{AS}$ & $0.825 \mathrm{BU}$ & & & \\
$5 / 6$ & $0.179 \mathrm{AS}$ & $0.188 \mathrm{BU}$ & $0.525 \mathrm{BU}$ & $0.652 \mathrm{AS}$ & $0.686 \mathrm{BU}$ & & & \\
$6 / 7$ & $0.150 \mathrm{AS}$ & $0.157 \mathrm{BU}$ & $0.578 \mathrm{AS}$ & & & & & \\
$3 / 5$ & $0.373 \mathrm{AS}$ & $0.393^{*}$ & $0.705 \mathrm{AS}$ & $0.730 \mathrm{AS}$ & $0.768 \mathrm{BU}$ & $0.815 \mathrm{BS} *$ & $0.820 \mathrm{AS}$ & \\
$5 / 7$ & $0.248 \mathrm{BS}$ & $0.251 \mathrm{AU}$ & $0.281 \mathrm{AS}$ & $0.518 \mathrm{BS}$ & $0.519 \mathrm{AU}$ & $0.592 \mathrm{BU}$ & $0.716 \mathrm{BU}$ & \\
$7 / 9$ & $0.175 \mathrm{BS}$ & $0.179 \mathrm{AU}$ & $0.228 \mathrm{AU}$ & $0.388 \mathrm{BS}$ & $0.394 \mathrm{AU}$ & $0.470 \mathrm{BU}$ & $0.560 \mathrm{AU}$ & $0.678 \mathrm{AU}$ \\
$4 / 7$ & $0.051 \mathrm{AS}$ & $0.064 \mathrm{BU}$ & $0.359 \mathrm{BS}$ & $0.369 \mathrm{AU}$ & $0.727 \mathrm{BU}$ & $0.808 \mathrm{AS}$ & $0.860 \mathrm{BU}$ & \\
$5 / 8$ & $0.050 \mathrm{AS}$ & $0.064 \mathrm{BU}$ & $0.293 \mathrm{BS}$ & $0.303 \mathrm{AU}$ & $0.640 \mathrm{BU}$ & $0.732 \mathrm{AS}$ & $0.738 \mathrm{BU}$ & \\
$7 / 10$ & $0.049 \mathrm{AS}$ & $0.064 \mathrm{BU}$ & $0.210 \mathrm{BS}$ & $0.222 \mathrm{AU}$ & $0.517 \mathrm{BU}$ & $0.565 \mathrm{BS}$ & $0.597 \mathrm{AU}$ & \\
\hline
\end{tabular}

where the position of the observed TNO's, taken from the Minor Planet Center table, is also indicated. We may notice that the distribution of objects does not exceed the eccentricity values of the periodic orbits at $e \approx 0.4$. Especially in the case of $2 / 3$ resonance, where the periodic orbits are located at $e \approx 0.47$, the distribution of objects shows an extensive spread up to $e \approx 0.35$. In Kotoulas and Voyatzis (2004) it is shown that for the planar elliptic problem and, particularly, for the resonances $2 / 3$ and $3 / 4$, regular orbits exist for eccentricity values almost up to the position of the unstable periodic orbits. The $4 / 7$ resonance does not possess stable periodic orbits for low and moderate values of the eccentricity, but, as it is shown in Fig. 2, a large number of objects is observed at low eccentricities.

\subsection{Bifurcations from planar circular RTBP to $3 D$ circular RTBP}

The three dimensional families of symmetric periodic orbits in the circular problem bifurcate from the vertical critical orbits of the corresponding planar circular problem (Hénon (1973)). The bifurcation points from the planar circular problem to the corresponding $3 \mathrm{D}$ one are presented in Fig.1b and in Table 2. The families consist of periodic orbits which are symmetric to $x z$-plane $(y(0)=\dot{x}(0)=\dot{z}(0)=0$, type A) or to $x$-axis $(y(0)=\dot{x}(0)=z(0)=0$, type B). For both symmetries, families with stable $(\mathrm{S})$ or 


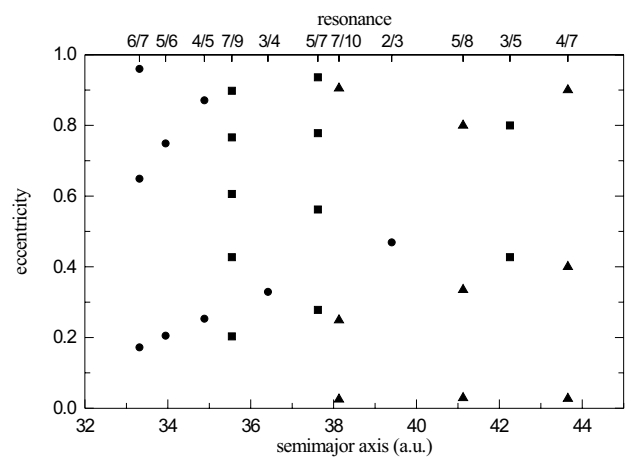

(a)

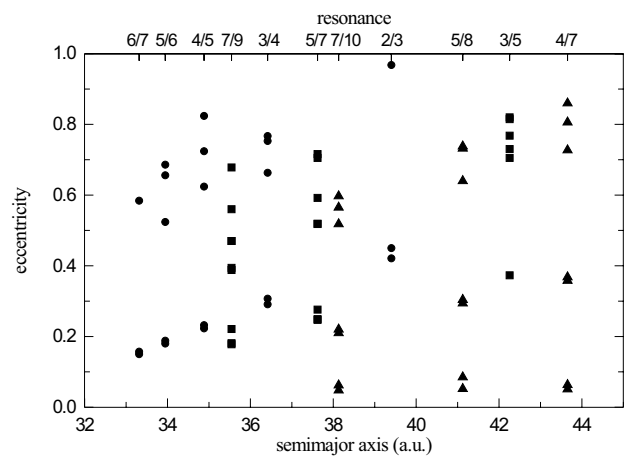

(b)

Figure 1. Bifurcation points a) from planar circular to planar elliptic problem b) from planar circular to 3D circular problem. Circles, squares and triangles refer to 1st, 2nd and 3rd order resonances, respectively.

unstable (U) periodic orbits exist. The multiplicity may change many times along the families. In Table 2 the type of symmetry and the stability of the bifurcating family is indicated. For the 1st order resonances the stability type does not change at least up to $i=20^{\circ}$. For the $3 / 5$ resonance some exceptions were found. From the bifurcation point at $e=0.393$ two families are generated; one has symmetry of type A and is stable (S) and the other one has symmetry of type B and is unstable. Moreover, the bifurcating families at $e=0.705$ and $e=0.815$ start as stable but they become doubly unstable for $i \approx 9^{\circ}$ and $i \approx 25^{\circ}$, respectively. We should remark that for $e<0.5$ all resonances have stable families which are of symmetry type A. Additionally, the second and third order resonances have families of stable orbits of symmetry type B too. A detailed study for the resonant cases $1 / 2,2 / 3$ and $3 / 4$ is given in Kotoulas and Hadjidemetriou (2002).

\section{Asymmetric resonances}

We recall that the asymmetric resonances are of the form $n / n^{\prime}=1 / q, q=2,3, \ldots$ and located beyond the 47 A.U. In all such resonances and in the framework of the planar circular RTBP, the family I presents the same characteristics as in the case of low or high-order resonances studied in the previous section. But along the family II a different structure is obtained. The family II of asymmetric resonances starts as stable; it becomes unstable at a certain value of eccentricity and then becomes again stable at a very high value of it. The critical points, where the stability changes, correspond to bifurcations to asymmetric periodic orbits (Voyatzis et al. (2004)). This scenario is valid for $1 / 2,1 / 3$, $1 / 4$ and $1 / 6$ mean motion resonances with Neptune. The eccentricity values of the above bifurcation points are given in Table 3 . An exceptional case is the $1 / 5$ resonance where a collision orbit is obtained along the family II. Asymmetric periodic orbits exist but their complete localization needs further investigation.

The bifurcation points from the planar circular to planar elliptic problem and the stability type of the generated families of periodic orbits are presented in Table 4 using the notation $\mathrm{Bf}_{e l} n$. Checking the vertical stability of periodic orbits of the corresponding planar circular problem, we found that there exist vertically critical orbits along the families of the resonances $1 / 2$ and $1 / 3$. These points are presented in Table 4 too and using the notation $\mathrm{Bf}_{3 D} n$. 


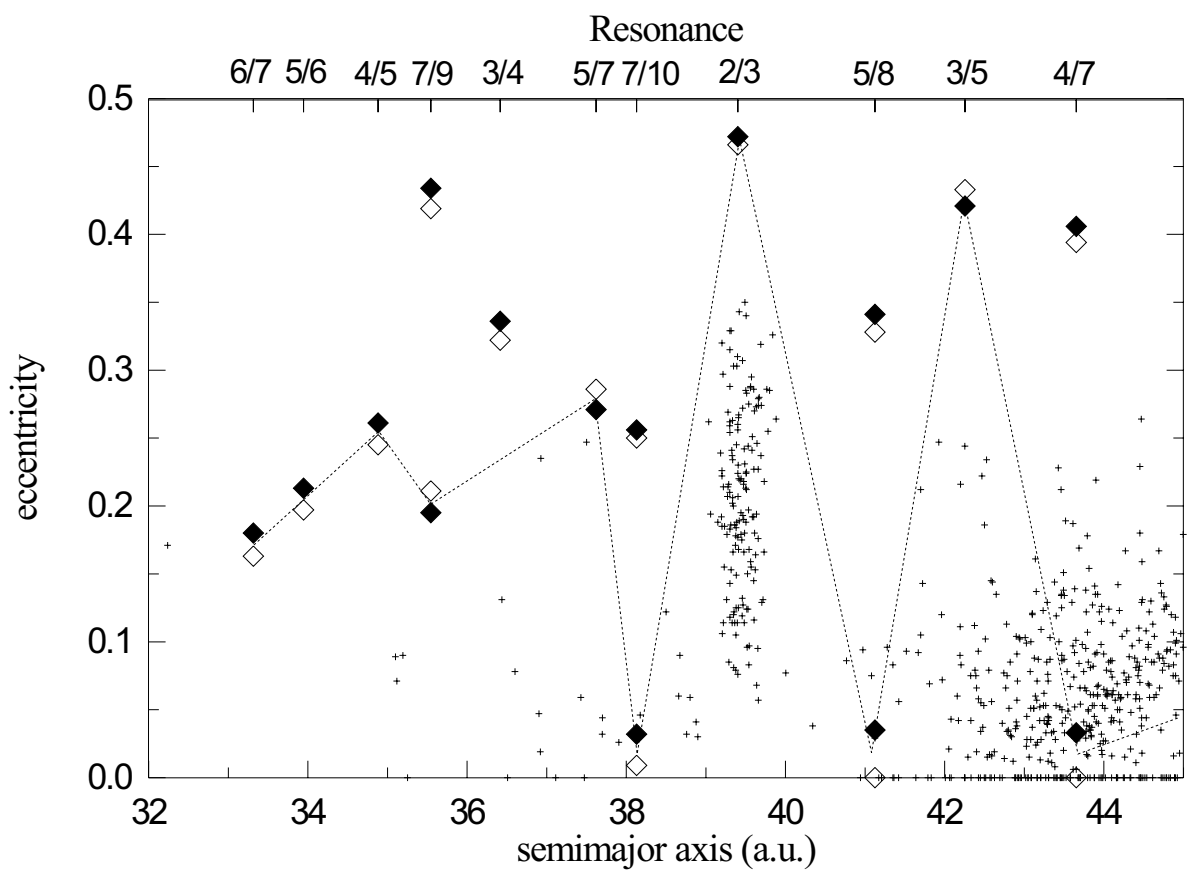

Figure 2. The distribution of TNOs on the $a-e$ plane and the position of the isolated periodic orbits of the planar elliptic problem for $e^{\prime}=0.00912$. Solid and empty symbols refer to the periodic orbits of the families $E_{p}$ and $E_{a}$ respectively.

Table 3. Eccentricity values of bifurcation points for asymmetric periodic orbits in the planar circular RTBP

\begin{tabular}{cccc}
\hline Resonance & $a_{\text {res }}$ (A.U.) & Bf1 & Bf2 \\
\hline $1 / 2$ & 47.78 & 0.035 & 0.960 \\
$1 / 3$ & 62.53 & 0.123 & 0.972 \\
$1 / 4$ & 75.75 & 0.201 & 0.978 \\
$1 / 5$ & 87.90 & $?$ & $\simeq 1.0$ \\
$1 / 6$ & 99.26 & 0.322 & 0.984 \\
\hline
\end{tabular}

Table 4. Eccentricity values of bifurcation points from the circular to the elliptic planar $\operatorname{RTBP}\left(\mathrm{Bf}_{e l} n\right)$ and from planar to 3D circular RTBP $\left(\mathrm{Bf}_{3 D} n\right)$

\begin{tabular}{ccccccc}
\hline Resonance & $\mathrm{a}(\mathrm{A} . \mathrm{U})$. & $\mathrm{Bf}_{e l} 1$ & $\mathrm{Bf}_{e l} 2$ & $\mathrm{Bf}_{e l} 3$ & $\mathrm{Bf}_{3 D} 1$ & $\mathrm{Bf}_{3 D} 2$ \\
\hline $1 / 2$ & 47.78 & $0.070 \mathrm{UU}$ & $0.637 \mathrm{UU}$ & & $0.059 \mathrm{AU}$ & $0.066 \mathrm{BU}$ \\
$1 / 3$ & 62.53 & $0.135 \mathrm{UU}$ & $0.759 \mathrm{UU}$ & $0.950 \mathrm{UU}$ & $0.112 \mathrm{AU}$ & $0.595 \mathrm{AS}$ \\
$1 / 4$ & 75.75 & $0.815 \mathrm{UU}$ & & & & \\
$1 / 5$ & 87.90 & & & & & \\
$1 / 6$ & 99.26 & $0.870 \mathrm{UU}$ & & & & \\
\hline
\end{tabular}

\section{Conclusions}

We studied the resonant families of periodic orbits and presented the bifurcation points from the planar circular to planar elliptic and to three-dimensional circular one for all the first, second and third order E-K belt resonances. In most cases of the elliptic problem the bifurcating families appear in pairs, one with stable orbits and one with unstable orbits. The stability type does not change up to $e^{\prime}=0.1$. The $3 \mathrm{D}$ circular problem shows 


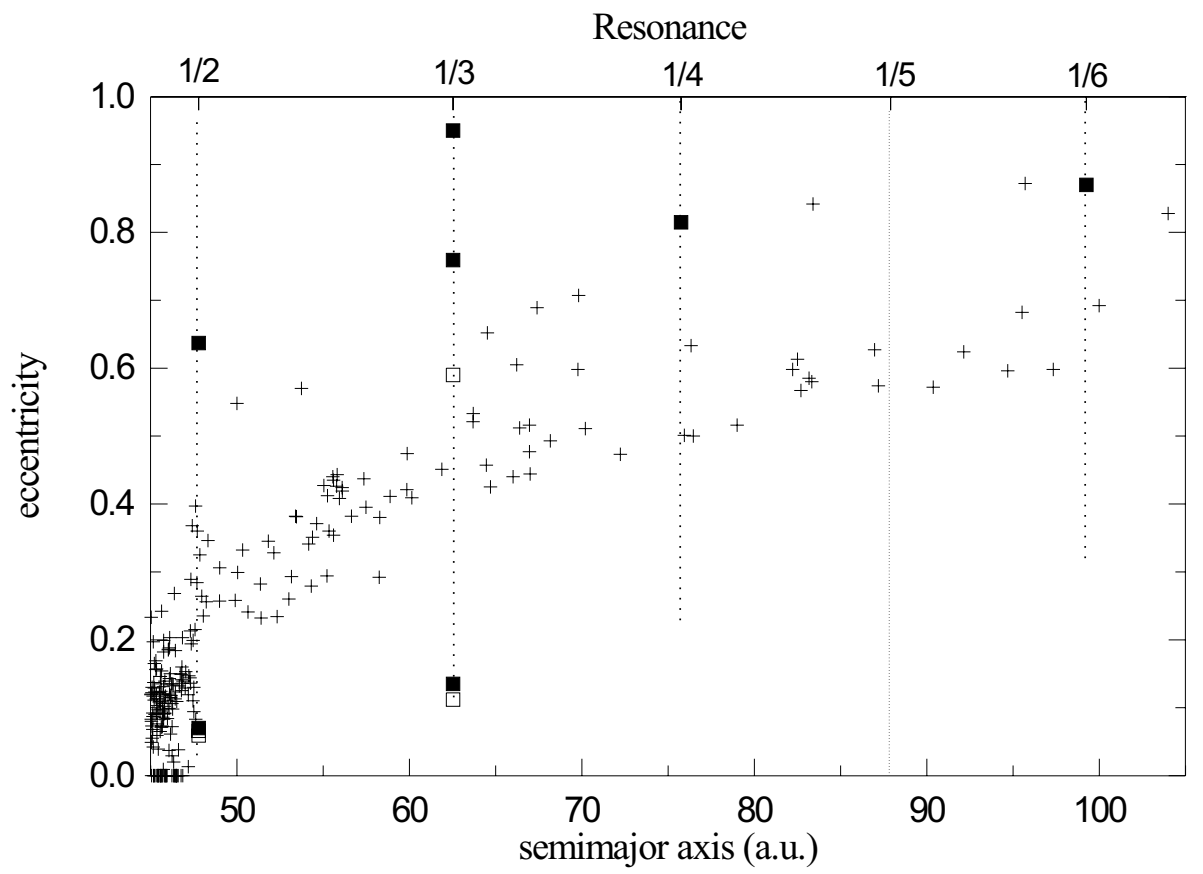

Figure 3. Bifurcation points and the distribution of TNOs at the region of the asymmetric resonances. Solid symbols refer to bifurcation points from the planar circular to the planar elliptic problem. Empty symbols refer to bifurcation points from the planar circular to the 3D circular problem. The dotted vertical lines indicate the eccentricity range where stable asymmetric periodic orbits can be found.

also both stable and unstable families. Generally, in this case, the families extend up to moderate or high inclination values preserving their stability type. The asymmetric resonances show bifurcation points from the planar circular to planar elliptic problem except the $1 / 5$ one. Bifurcations from the planar circular to the three-dimensional circular problem were found only for the cases $1 / 2$ and $1 / 3$.

\section{Acknowledgements}

We would like to acknowledge the research program "EPEAEK II, PYTHAGORAS No.21878" of the Greek Ministry of Education and E.U., which supported the present work.

\section{References}

Hadjidemetriou, J.D. and Ichtiaroglou, S. 1984, Astron. Astrophys. 131, 20

Hénon, M. 1973, Astron. Astrophys. 28, 415

Holman M.J. and Wisdom J. 1993, Astron. J. 105, 1987

Knežević Z., Milani A., Farinella P., Froeschlé Ch. and Froeschlé C. 1991, Icarus 93, 316

Kotoulas T. and Hadjidemetriou, J.D. 2002, Earth, Moon, Planets 91, 63

Kotoulas T. and Voyatzis G. 2004, Cel. Mech. Dyn. Astron. 88, 343.

Levison, H.F. and Duncan, M.J. 1993, Astrophys. J. 406, L35

Malhotra, R. 1996, Astron. J. 111, 540

Morbidelli, A. 1999, Cel. Mech. Dyn. Astr. 72, 129

Morbidelli, A., Brown, M.E. and Levison, H.F 2003, Earth, Moon, Planets 92, 1

Voyatzis G., Kotoulas T. and Hadjidemetriou J.D. 2004, Cel. Mech. Dyn. Astr., in press. 